\title{
ERRATUM
}

Pramana - J. Phys., Vol. 51, Nos. 3 \& 4, September/October 1998, pp. 317-355

\section{Charges in Gauge Theories}

by ROBIN HORAN, MARTIN LAVELLE and DAVID MCMULLAN.

- On p. 327 just before Eq. 39 it should read $\beta=-1$.

- On p.328 it should read:

The free field equation $\square A_{\mu}^{\text {free }}=0$ implies that

$$
A_{\mu}^{\mathrm{free}}(x)=\int d^{3} z\left(\partial_{0}^{z} D(x-z) A_{\mu}^{\mathrm{free}}(z)-D(x-z) \partial_{0} A_{\mu}^{\text {free }}(z)\right)
$$

where $D(x-z)$ is the commutator function for free fields:

$$
D(x-y)=-\int \frac{d^{3} \mathbf{k}}{(2 \pi)^{3}} \frac{1}{\omega_{k}} e^{i \mathbf{k} \cdot(\mathbf{x}-\mathbf{y})} \sin \left(\omega_{k}\left(x^{0}-y^{0}\right)\right) .
$$

The identification in (48) is made by first observing that the right hand side is independent of $z^{0}$ : setting $z^{0}=x^{0}$ then implies the result. Exploiting this $z^{0}$-independence, the commutator $\left[A_{\mu}^{\text {free }}(x), A_{\nu}^{\text {free }}(y)\right]$ is simply calculated by using (48) with $z^{0}=y^{0}$. Then the equal time commutation relations $\left[A_{\mu}(y), \dot{A}_{\nu}(z)\right]_{\text {et }}=-i g_{\mu \nu} \delta(y-z)$ can be used. This, in conjunction with our observation that the free and asymptotic interacting fields have the same commutators, results in the space time commutators in Feynman gauge being:

$$
\left[A_{\mu}(x), A_{\nu}(y)\right]=\left[A_{\mu}^{\mathrm{free}}(x), A_{\nu}^{\mathrm{free}}(y)\right]=-i g_{\mu \nu} D(x-y)
$$

- On p. 340 , Eq. 93 should read:

$$
F_{R}=\frac{e^{2}}{(2 \pi)^{3}} \int d^{4} k e^{i k \cdot x} \theta\left(k_{0}\right) \delta\left(k^{2}\right) \frac{r_{\mu} r_{\nu}}{(r \cdot k)^{2}} \Pi_{R}^{\mu \nu}
$$

(corrections in references)

[9] S A Gogilidze, A M Khvedelidze, D M Mladenov, and H P Pavel, Phys. Rev. D57, 7488 (1997), hep-th/9707136

[11] J Fröhlich, G Morchio, and F Strocchi, Phys. Lett. B89, 61 (1979)

[23] T Kawai and H P Stapp, Phys. Rev. D52, 2505 (1995), quant-ph/9511031

[33] N N Bogolyubov and D V Shirkov, Introduction to the Theory of Quantised Fields, 3rd Edition, (Wiley-Interscience, New York, 1980)

[36] E Bagan, M Lavelle, and D McMullan, Phys. Rev. D57, 4521 (1998), hep-th/9712080

[60] R G Stuart, in Perspectives for Electroweak Interactions in $e^{+} e^{-}$Collisions, Ed. B A Kniehl, (World Scientific, Singapore, 1995), hep-ph/9504308 